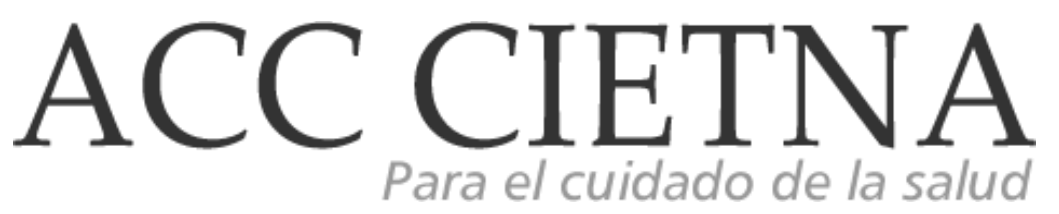

\title{
Funcionalidad familiar y autocuidado en personas con diabetes mellitus tipo 2 en el Policlínico Manuel Manrique Nevado, Chiclayo 2019
}

\section{Family and self-care functionality in people with type 2 diabetes mellitus in the Polyclinic Manuel Manrique Nevado, Chiclayo 2019}

\author{
Fatima Milagros Campoverde-Vilanueva *, 1 , \\ 2, a \\ fatimamil34@gmail.com \\ https://orcid.org/0000-0001-8741-8706
}

Iliana del Rosario Muro-Exebio ${ }^{1,3, ~ a, ~ b, ~ c ~}$ imuro@usat.edu.pe

https://orcid.org/0000-0003-3964-4113

\footnotetext{
* Autor corresponsal

1 Escuela de Enfermería, Facultad de Medicina, Universidad Católica Santo Toribio de Mogrovejo, Chiclayo, Perú

${ }^{2}$ Trabajador independiente
}

\author{
3 Servicio de Neonatología, Hospital Regional Docente \\ Las Mercedes, Chiclayo, Perú \\ a Licenciada en Enfermería \\ b Magister en Gerencia de Servicios de Salud \\ c Especialista en Cuidado de Enfermería al Niños y \\ Adolescente y Enfermería Comunitaria
}

\section{Fechas importantes}

Recibido: 2021-08-07

Aceptado: 2021-11-15

Publicado online: 2021-12-17

\section{Resumen}

Objetivo: Determinar la relación entre la funcionalidad familiar y el autocuidado en las personas con Diabetes Mellitus Tipo 2 en el Policlínico Manuel Manrique Nevado, Chiclayo 2019. Metodología: La investigación fue cuantitativa con diseño transversal y correlacional. Tuvo como objetivo determinar la relación entre la funcionalidad familiar y el autocuidado en las personas con Diabetes Mellitus Tipo 2. La información se recolectó mediante los instrumentos FACES III (Funcionalidad familiar) y SDSCA (Autocuidado en Diabetes), aplicado a 84 personas conformaron la muestra censal. Se implementó una prueba piloto para probar la confiabilidad de los instrumentos, para lo cual se utilizó Alfa de Crombrach obteniendo 0.79 y 0.72 respectivamente. La relación entre las variables se probó mediante Coeficiente de Correlación de Spearman. Resultados principales: Predominó la funcionalidad de rango medio (52.4\%) y la categoría sin prácticas de autocuidado en la persona con DM tipo 2 (86.9\%). La funcionalidad familiar tuvo relación significativa con el autocuidado en las personas con DM tipo 2 solo en su dimensión toma de medicamentos $(p=0.034)$. Conclusiones: De manera global no existe relación significativa entre la funcionalidad familiar y el autocuidado en las personas con Diabetes Mellitus Tipo 2 en el Policlínico Manuel Manrique Nevado, Chiclayo 2019. Recomendaciones: Fomentar actividades 
que incluyan al paciente diabético y su familia además concientizar a la población para que tomen conciencia sobre la Diabetes para poder prevenirla.

Palabras clave: Familia (D005190), autocuidado (D012648), diabetes mellitus (D003920)

\begin{abstract}
Objective: To determine the relationship between family functionality and self-care in people with Type 2 Diabetes Mellitus in the Manuel Manrique Nevado Polyclinic, Chiclayo 2019. Methodology: The research was quantitative with a cross-sectional and correlational design. Its objective was to determine the relationship between family functionality and self-care in people with Type 2 Diabetes Mellitus. The information was collected using the instruments FACES III (Family Functionality) and SDSCA (Self-care in Diabetes), applied to 84 people made up the sample census. A pilot test was implemented to test the reliability of the instruments, for which Crombrach's Alpha was used, obtaining 0.79 and 0.72 respectively. The relationship between the variables was tested using Spearman's Correlation Coefficient. Main results: Mid-range functionality (52.4\%) and the category without self-care practices predominated in the person with type 2 DM $(86.9 \%)$. Family functionality had a significant relationship with self-care in people with type $2 \mathrm{DM}$ only in its medication-taking dimension $(p=0.034)$. Conclusions: Overall, there is no significant relationship between family functionality and self-care in people with Type 2 Diabetes Mellitus in the Manuel Manrique Nevado Polyclinic, Chiclayo 2019. Recommendations: Promote activities that include the diabetic patient and his family, as well as raise awareness among the population so that they become aware of Diabetes in order to prevent it.
\end{abstract}

Keywords: Family (D005190), self-care (D012648), diabetes mellitus (D003920)

\title{
Introducción
}

Actualmente, a nivel mundial la Diabetes Mellitus tipo 2 es uno de los desafíos de salud pública que va en incremento durante los últimos reportes además es una iniciativa global para la prevención y el cuidado de esta enfermedad ${ }^{1}$. La Organización Mundial de la Salud (OMS) existían 420 millones de personas mayores de 21 años con Diabetes Mellitus en el año 2014. Los porcentajes más altos pertenecieron a las regiones del Pacífico Occidental, Sudoriental y Asia; donde se localizan cerca de la mitad de los casos en India, China y Estados Unidos².

La Federación Internacional de Diabetes (FID) estima América existe 21.7 a 31.9 millones de personas de la población adulta con Diabetes en el 2017. La Diabetes Mellitus representa el $95 \%$ de los casos a escala mundial donde su incidencia varia significativamente en Uruguay, Argentina, Brasil, Paraguay y Perú ${ }^{3}$.

El fomentar el autocuidado en las personas estadounidenses con Diabetes Mellitus tipo 2 tiene fuerte evidencia de mejorar la Hba1c (hemoglobina glicosilada), la calidad de vida, baja de peso, afrontamiento saludable, aumento de medidas preventivas y disminución de complicaciones agudas. El abordaje de esta enfermedad se ha modificado a una visión más centrada al paciente y ubica al individuo que vive con diabetes y a su familia en el epicentro del modelo de cuidado y atención de salud, trabajando en conjunto con el resto del equipo de salud, así lo revelan estudios realizados en Estados Unidos ${ }^{4}$.

En un estudio en Lima se afirma que el apoyo familiar a las personas adultas con Diabetes Mellitus, 64,6\% fue adecuado frente a las medidas de control, indicaciones médicas y apoyo emocional. Consecuentemente $52,1 \%$ estas personas evidencian comportamientos de autocuidado adecuados en el control metabólico, plan nutricional, ejercicios físicos, cuidado de pies y control farmacológico ${ }^{16}$. Sin embargo, en otro estudio en el mismo contexto revela que al adulto no le es posible realizar un autocuidado óptimo en cuanto al ejercicio, 
alimentación, higiene ya que se requiere incentivar a la familia en las tres dimensiones en lo emocional, social y física para el aporte del cambio de vida saludable de la persona diabética 5 .

En Lambayeque la prevalencia de Diabetes mellitus tipo 2 es del $54,3 \%$ en las personas de 30 a 60 años, los cuales $92 \%$ son procedentes de la costa además $42 \%$ presenta sobrepeso y obesidad $^{6}$. En un estudio en Chiclayo, se encontró que $38.9 \%$ de las pacientes adultas con Diabetes Mellitus tipo 2 tuvieron mayor frecuencia de autocuidados incorrectos en cuidados de los pies?.

Por otro lado, el Policlínico Manuel Manrique Nevado cuenta con un programa de Adulto/Adulto mayor que durante las prácticas realizadas en este establecimiento se pudo evidenciar que gran parte de los pacientes inscritos en el programa son mujeres. Con respecto al autocuidado de las personas diabéticas se observó que se olvidan tomar sus medicamentos, algunos pacientes no se adaptan al tratamiento farmacológico ya que les resulta difícil estar tomando medicamentos cada cierta hora. $Y$ con respecto al entorno familiar de la persona diabética se pudo observar que mayor parte de las familias no los ayuda a la compra de los alimentos idóneos para el cumplimiento de la dieta, no les ofrece opciones para realizar ejercicios en familia así mismo una pequeña proporción de estos pacientes manifestaron que no cuentan con un familiar que los apoyen con los comportamientos para llevar una vida saludable.

Finalmente, bajo esta temática se puede deducir que la funcionalidad familiar es preponderante en el autocuidado de las personas diabéticas, sin embargo, no está claro que la funcionalidad familiar sea una fuente de apoyo para que la persona lleve un autocuidado por lo tanto es importante identificar si el apoyo de la familia y el autocuidado influyen en la persona diabética o alguna de las dos es más decisiva que la otra ${ }^{8}$. Frente a esta realidad se formuló la siguiente interrogante: ¿Cuál es la relación entre la funcionalidad familiar y el autocuidado de las personas con Diabetes Mellitus Tipo 2 - ¿Policlínico Manuel Manrique Nevado, Chiclayo 2019?

Por todo lo expuesto se planteó como objetivo general determinar la relación de la funcionalidad familiar y el autocuidado en las personas con Diabetes Mellitus Tipo 2 - Policlínico Manuel Manrique Nevado. Chiclayo, 2019 así como objetivos específicos: describir la funcionalidad familiar de las personas con Diabetes Mellitus Tipo 2 e identificar el autocuidado de las personas con Diabetes Mellitus Tipo 2 en el Policlínico Manuel Manrique Nevado, Chiclayo 2019.

El estudio de investigación se justificó puesto que la Diabetes Mellitus tipo 2 es una condición que va en incremento en la etapa adulta en nuestro país, pudiendo llegar a tener carácter epidemiológico, es además una enfermedad que tiene complicaciones de alto impacto para las personas pues merman su calidad de vida y para las instituciones de salud generan altos costos de la atención que se brinda a estas complicaciones. La familia puede constituirse en una fuente de apoyo para el autocuidado de la persona con Diabetes Mellitus porque mejora la adherencia al tratamiento por lo que se justifica investigar en el contexto del Policlínico Manuel Manrique Nevado, la relación que existe entre la funcionalidad de los familiares y el autocuidado de las personas que presentan Diabetes Mellitus tipo $2^{9}$. Por otro lado, se justificó investigar toda vez que existe poca evidencia con enfoque cuantitativo relacionadas con la temática.

En el ámbito de la profesión de Enfermería los resultados de la investigación permitieron ampliar información sobre el rol que ocupa la familia en la generación y sostenibilidad del 
autocuidado de la persona con Diabetes Mellitus Tipo 2. Para las instituciones formadoras de recursos humanos en Enfermería, los resultados proporcionaron información útil acerca de la funcionalidad familiar y el autocuidado de la persona con Diabetes Mellitus que pueden incluir en las asignaturas relacionadas con el Adulto.

\section{Material y métodos}

La investigación encuadra en el tipo cuantitativo y el diseño fue no experimental, transversal y correlacional. La población estuvo compuesta con 84 personas adultas diabéticas atendidas en el Policlínico "Manuel Manrique Nevado" durante el 2018, cuyas edades oscilan entre 30 a 59 años. La muestra fue de tipo censal y el muestreo fue intencional ${ }^{10}$ La técnica utilizada en el estudio fue la encuesta, los instrumentos utilizados fueron 2 cuestionarios. Para medir la funcionalidad familiar se utilizó FACES III y el autocuidado de las personas con Diabetes Mellitus tipo 2 se midió a través del cuestionario SDSCA. El primero consta de 20 preguntas agrupadas en dos dimensiones: Cohesión familiar y Adaptabilidad o flexibilidad familiar y utiliza una escala tipo Likert que emplea una puntuación de 1 a 5 . La escala de Cohesión y Adaptabilidad familiar ha propiciado que las familias se clasifiquen en tres categorías: Equilibradas/Balanceadas, rango medio y extremas ${ }^{11}$.

El segundo instrumento SDSCA consta de 18 ítems los cuales se relaciona con las dimensiones establecidas; para su puntuación emplea una escala de 0 a 7 que equivale al número de días que la persona ha llevado a cabo un determinado comportamiento, consignado en cada ítem durante la última semana. La valoración de la encuesta estuvo catalogada como "sin prácticas de autocuidado" cuando sea < de 5 días y "con prácticas de autocuidado" cuando sea > de 5 días ${ }^{12}$.

Para la aplicación de los cuestionarios se obtuvo la autorización de EsSalud previa aprobación del proyecto de investigación con el NIT: 1298- 2018-4583, tras lo cual el director del Policlínico Manuel Manrique Nevado, la coordinadora de enfermeras y la enfermera encargada del Programa Adulto/Adulto Mayor brindando las facilidades correspondientes procediéndose a la ejecución de la investigación. La aplicación de los instrumentos planteados en la investigación se llevó a cabo entre Mayo y Junio del 2019, en la sala de espera del policlínico durante toda la mañana. Se empezó dando a conocer los objetivos y propósito de la investigación a los adultos en el momento que acudían a solicitar su atención en el programa, después de obtener la aceptación para participar en el estudio se procedió al llenado de los cuestionarios tomándose aproximadamente 25 minutos.

Los datos recolectados fueron organizados en una base de datos con el apoyo del programa Excel 2013 para luego ser transferidos al programa estadístico SPSS versión 25 que posteriormente permitió la elaboración de tablas estadísticas correspondientes donde se presentó información en frecuencias absolutas y relativas. Para comprobar si existió correlación significativa entre las variables se aplicó la prueba Spearman.

\section{Resultados}

Se presentó resultados sobre las características generales de las personas encuestadas donde el $61.9 \%$ de los entrevistados tuvieron entre 50 a 59 años. Respecto a las variables sociodemográficas más frecuentes: $66.7 \%$ fueron de sexo femenino; $69 \%$ tuvieron algún tipo de unión; $74 \%$ tuvieron un grado de instrucción de secundaria y superior, el $55.7 \%$ tuvieron 
algún tipo de empleo, el $60.7 \%$ tienen menos de 5 años con Diabetes Mellitus tipo 2 seguido del $82.1 \%$ utilizan antidiabéticos orales.

TABLA No 01: CARACTERISTICAS GENERALES DE LAS PERSONAS CON DIABETES MELIITUS TIPO 2 - POLICLINICO MANUEL MANRIQUE NEVADO. CHICLAYO, 2019.

\begin{tabular}{|c|c|c|c|}
\hline Caracteristicas generales & & $\mathrm{N}$ & $\%$ \\
\hline \multirow{3}{*}{ EDAD } & De 30 - 39 años & 11 & 13.1 \\
\hline & De 40 - 49 años & 21 & 25 \\
\hline & De 50 - 59 años & 52 & 619 \\
\hline \multirow{2}{*}{ SEXO } & Femenino & 56 & 66.7 \\
\hline & Masculino & 28 & 33.3 \\
\hline \multirow{4}{*}{ ESTADO CIVIL } & Casado & 45 & 53.6 \\
\hline & Conviviente & 12 & 15.5 \\
\hline & Divorciado & 1 & 1.2 \\
\hline & Soltero & 25 & 29.8 \\
\hline \multirow{4}{*}{ EDUCACION } & Ninguno & 2 & 2.4 \\
\hline & Primaria & 19 & 226 \\
\hline & Secumdaria & 45 & 53.6 \\
\hline & Superior & 18 & 21.4 \\
\hline \multirow{3}{*}{ OCUPACION } & Ama de casa & 34 & 40.5 \\
\hline & Desempleado & 3 & 3.6 \\
\hline & Empleado & 43 & 559 \\
\hline \multirow{3}{*}{ ANOS CON DM TIPO } & $<5$ aั่ง & 51 & 60.7 \\
\hline & $5-10$ สักัด & 25 & 29.8 \\
\hline & $>10$ בที०s & 8 & 9.5 \\
\hline \multirow{4}{*}{ MEDICACION } & Anticiabéticos Orales & 69 & 82.1 \\
\hline & Anticliabéticos Orales e Insulina & 6 & 7.1 \\
\hline & Insulina & 9 & 10.7 \\
\hline & Total & 84 & $100 \%$ \\
\hline
\end{tabular}

\section{Interpretación:}

En la tabla $\mathrm{N}^{\circ} 02$ menciona que el $52.4 \%$ de las personas encuestadas presentó funcionalidad familiar de rango medio seguido del $23.8 \%$ que presentó funcionalidad familiar balanceada y extremo.

\begin{tabular}{|c|c|c|}
\hline FUNCIONALIDAD FAMILIAR & $\mathrm{N}$ & $\%$ \\
\hline Balanceada & 20 & 23.8 \\
\hline Extremo & 20 & 23.8 \\
\hline Rango Medio & 44 & 52.4 \\
\hline Total & 84 & 100.0 \\
\hline
\end{tabular}

\section{Interpretación:}

En la tabla $\mathrm{N}^{\circ} 03$ se evidencio que el $86.9 \%$ de las personas encuestadas fueron calificadas "sin prácticas de autocuidado" seguido $13.1 \%$ fueron calificadas "con prácticas de autocuidado". 
TABLA N $N^{\circ}$ 03: AUTOCUIDADO DE LAS PERSONAS CON DIABETES MELLITUS TIPO 2 - POLICLINICO MANUEL MANRIQUE NEVADO. CHICLAYO, 2019.

\begin{tabular}{|c|c|c|c|c|}
\hline \multirow{2}{*}{ AUTOCUIDADO } & \multicolumn{2}{|c|}{ Sin autocuidado } & \multicolumn{2}{|c|}{ Con autocuidado } \\
\hline & $\mathrm{N}$ & $\%$ & $\mathrm{~N}$ & $\%$ \\
\hline Dieta & 75 & 89.3 & 9 & 10.7 \\
\hline Actividad fisica & 15 & 17.9 & 69 & 82.1 \\
\hline Análisis de Sangre & 68 & 81 & 16 & 19 \\
\hline Cuidados de los pies & 3 & 3.6 & 81 & 96.4 \\
\hline Medicamentos & 5 & 6 & 79 & 94 \\
\hline Tabaquismo & 67 & 79.8 & 17 & 20.2 \\
\hline Total & 73 & 86.9 & 11 & 13.1 \\
\hline
\end{tabular}

\section{Interpretación:}

En la tabla $\mathrm{N}^{\circ} 04$ se muestra que de manera global existe una correlación positiva débil $($ Rho $=0.096)$ entre la funcionalidad familiar y el autocuidado; sin embargo, esta relación no es significativa. Por tanto, se acepta la H0: No existe relación significativa entre la funcionalidad familiar y el autocuidado en las personas con Diabetes Mellitus Tipo 2 - Policlínico Manuel Manrique Nevado. Chiclayo, 2019. Sin embargo, se evidencia que existe una relación significativa entre el funcionamiento familiar y el autocuidado en cuanto a la dimensión de medicamentos en la persona con DM tipo $2(p=0.034)$ con una correlación positiva media $(\mathrm{Rho}=0.201)$

TABLA N $N^{\circ}$ 04: FUNCIONAIIDAD FAMILIAR Y AUTOCUIDADO DE PERSONAS CON DIABETES MELLITUS TIPO 2 - POLICLINICO MANUEL MANRIQUE NEVADO. CHICLAYO, 2019

\begin{tabular}{|c|c|c|c|c|c|c|c|c|c|}
\hline \multirow{3}{*}{$\begin{array}{c}\text { AUTOCUIDADO - } \\
\text { SDSCA }\end{array}$} & \multicolumn{6}{|c|}{ FUNCIONAILIDAD FAIMILIAR-FACES III } & & & \multirow{3}{*}{$\begin{array}{l}\text { Valor } \\
\text { de } P\end{array}$} \\
\hline & \multicolumn{2}{|c|}{ BAI_AINCEADA } & \multicolumn{2}{|c|}{ EXTREMOO } & \multicolumn{2}{|c|}{ RANGO MIEDIO } & \multicolumn{2}{|c|}{ TOTAI } & \\
\hline & $\mathbf{N}$ & $\%$ & $\mathrm{~N}$ & $\%$ & $\mathbf{N}$ & $\%$ & $\mathrm{~N}$ & $\%$ & \\
\hline $\begin{array}{c}\text { DIETA } \\
\text { BAIANCEADA }\end{array}$ & & & & & & & & & \multirow[b]{3}{*}{0.468} \\
\hline Sin autocuidado & 18 & 21.4 & 19 & 22.6 & 38 & 45.2 & 75 & 89.3 & \\
\hline Con autocuidado & 2 & 2.4 & 1 & 1.2 & 6 & 7.1 & 9 & 10.7 & \\
\hline \multicolumn{10}{|l|}{ ACTIVIDAD FISICA } \\
\hline Sin autocuidado & 4 & 4.8 & 3 & 3.6 & 8 & 9.5 & 15 & 17.9 & \multirow[b]{2}{*}{0.480} \\
\hline Con autocuidado & 16 & 19.0 & 17 & 20.2 & 36 & 42.9 & 69 & 82.1 & \\
\hline \multicolumn{10}{|l|}{$\begin{array}{l}\text { ANAIISIS DE } \\
\text { SANGRE }\end{array}$} \\
\hline Sin autocuidado & 17 & 20.2 & 13 & 15.5 & 38 & 45.2 & 68 & 81.0 & \multirow[b]{2}{*}{0.228} \\
\hline Con autocuidado & 3 & 3.6 & 7 & 8.3 & 6 & 7.1 & 16 & 19.0 & \\
\hline \multicolumn{10}{|l|}{$\begin{array}{l}\text { CUIDADOS DE LOS } \\
\text { PIES }\end{array}$} \\
\hline Sin autocuidado & 0 & 0 & 1 & 1.2 & 2 & 2.4 & 3 & 3.6 & \multirow{2}{*}{0.231} \\
\hline Con autocuidado & 20 & 23.8 & 19 & 22.6 & 42 & 50 & 81 & 96.4 & \\
\hline \multicolumn{10}{|l|}{ MEDICAMENTOS } \\
\hline Sin autocuidado & 3 & 3.6 & 1 & 1.2 & 1 & 1.2 & 5 & 6.0 & \multirow{2}{*}{0.034} \\
\hline Con autocuidado & 17 & 20.2 & 19 & 22.6 & 43 & 51.2 & 79 & 94.0 & \\
\hline \multicolumn{10}{|l|}{ TABAQUISMO } \\
\hline Sin autocuidado & 5 & 6.0 & 2 & 2.4 & 10 & 11.9 & 17 & 20.2 & \multirow{2}{*}{0.423} \\
\hline Con autocuidado & 15 & 17.9 & 18 & 21.4 & 34 & 40.5 & 67 & 79.8 & \\
\hline TOTAL & 20 & 23.8 & 20 & 23.8 & 44 & 52.4 & 84 & 100 & \\
\hline
\end{tabular}




\section{Discusión}

A lo largo de la vida, la familia es un sostén importante para la persona con Diabetes y en torno a ella además cuando sospecha una crisis por lo tanto conlleva un proceso de aceptación y adaptación en relación al bienestar físico, emocional y mental de la persona diabética pero también debe incluirse eficazmente en el proceso de entender la enfermedad y la forma de emanar en cada situación y su nuevo estilo de vida ${ }^{13}$.

En la Tabla $\mathrm{N}^{\circ} 02$ se consigna la información acerca de la funcionalidad familiar de las personas adultas diabéticas encuestadas, encontrándose que $23.8 \%$ tuvieron un funcionamiento balanceado, $23.8 \%$ extremo y $52.4 \%$ rango medio debido a tener una familia catalogada como unida respecto a la cohesión familiar, lo cual implica que son capaces de equilibrar en situaciones complicadas como la Diabetes Mellitus tipo 2 y al mismo tiempo por tener una familia calificada como caótica, lo cual implica que las decisiones tomadas de manera impulsivas y muy cambiantes en los roles como integrantes de la familia.

Los resultados son similares a los reportados por Vásquez ${ }^{13}$ quien reporta $64.4 \%$ tuvieron funcionamiento familiar de rango medio y $35.6 \%$ tuvieron funcionabilidad familiar extrema también con Zambrano ${ }^{14}$ quien reporta $40.7 \%$ tuvieron familias medianamente equilibradas (rango medio), $24.7 \%$ familias equilibradas (balanceadas) $34.7 \%$ familias extremas. $Y$ Moraga ${ }^{15}$ quien reporta que $48.5 \%$ son de nivel de funcionamiento familiar percibido es de rango medio, $23.9 \%$ funcionamiento familiar extremo y $5.8 \%$ funcionamiento familiar balanceado.

En la presente investigación se pudo comprobar el predominio de las familias con funcionamiento familiar de rango medio lo que implica que su funcionalidad familiar presenta dificultades en la dimensión de cohesión o adaptabilidad, las cuales pueden ser presentadas por circunstancias que pasa la familia en un momento, lo cual refleja la existencia de una relación entre sus miembros, así como también una capacidad de obstrucción ante los diversos cambios recurrentes que puede sufrir una familia a lo largo de su ciclo vital ${ }^{16}$.

En la funcionalidad familiar de rango medio influye la cohesión o la adaptabilidad, cuando predomina la cohesión en un nivel moderado implica que la toma de decisiones se realiza sin dejar de lado sus objetivos personales y son familias autoritarias, generalmente tiene un liderazgo parenteral autoritariamente fuerte y presenta la falta de evolución al cambio dentro del núcleo familiar. En la adaptabilidad de las familias de rango medio se caracteriza por una falta de organización, los roles son cambiantes frecuentemente y las decisiones son de forma impulsivas. Además, la familia de rango medio mantiene un equilibrio saludable de poderes donde las reglas se pueden cambiar ocasionalmente ${ }^{17}$.

En la tabla $\mathrm{N}^{\circ} 03$ se puede observar que $86.9 \%$ de las personas encuestadas fueron catalogadas como "sin autocuidado" y $13.1 \%$ como "con autocuidado". Lo cual podría deberse a que refirieron consumir algún tipo de postres todos los días además de frutas y verduras algunos días a la semana. Los resultados son similares a los reportados por Del Pezo ${ }^{18}$ quien reportó que $70 \%$ no siguen su plan de alimentación y $28.5 \%$ consumen una dieta hipograsa e hiposódica. Pero diferentes a León ${ }^{19}$ quien reporto que $53.4 \%$ de las personas diabéticas llevan un plan alimentario saludable de 3 a 5 días durante los últimos 7 días y Amaral $^{20}$ reportando que $83.8 \%$ de personas adultas con Diabetes llevan una dieta libre de azúcar, dulces y pastas. 
Se pudo evidenciar preponderancia de personas diabéticas en el Policlínico Manuel Manrique Nevado que no tienen prácticas de autocuidado en la alimentación evidenciando un incremento en el consumo de grandes cantidades de carbohidratos y grasas lo cual podría condicionar que la persona diabética no alcance la disminución del peso corporal deseado, además de tener riesgo de altos valores glucémicos postprandiales. Así mismo, puede tener poca probabilidad de controlar eficazmente las alteraciones metabólicas afectándose de manera directa al organismo lo que puede provocar enfermedades agregadas insuficiencia renal, insuficiencia cardiaca e incluso la Hipertensión arterial ${ }^{21}$

En cuanto al autocuidado de la actividad física se encontró que $82.1 \%$ fueron considerados como "con prácticas de autocuidado" debido a que refirieron realizar caminatas de 30 minutos por día; además de realizar ejercicios específicos (aeróbicos). Los resultados encontrados son similares a los reportados por Del Pezo ${ }^{18}$ quien reportó que $57.1 \%$ realizan caminatas seguido del $17.1 \%$ no realizan ningún tipo de ejercicios y a los resultados encontrados por Galiano ${ }^{22}$ mencionando que $60.2 \%$ de las personas diabéticas realizaron ejercicios al menos 5 veces al día y $39.8 \%$ no realizaron ningún tipo de ejercicio. Así mismo fueron disimiles a los reportados por Jaimes ${ }^{23}$ quien reporta que $71 \%$ de los pacientes diabéticos nunca realizaron actividad física y $29 \%$ si lleva un control de actividad física diaria.

El predominio de las personas con Diabetes mellitus tipo 2 del Policlínico Manuel Manrique Nevado que realizan alguna actividad física hace que tengan un mejor estilo de vida y por lo tanto reduzcan complicaciones posteriormente. La actividad física es un elemento importante para el mejoramiento de su salud de las personas encuestadas lo cual trae beneficios fisiológicos y psicológicos, la reducción de su peso, el control de su glucosa y la reducción de estrés. La realización de actividad física en estas personas son al menos 30 minutos por día haciendo actividades en casa o en el trabajo además que refirieron hacer aeróbicos sea en gimnasio o en casa.

Con respecto al autocuidado frente a los análisis de sangre se encontró que $81 \%$ fueron catalogadas como "sin prácticas de autocuidado" con respecto a los controles de glucosa. Los resultados encontrados son similares a los reportados por Pérez ${ }^{24}$ reportó que $61.9 \%$ de personas con Diabetes presentaron glucosa descontrolada. Igualmente, los resultados reportados por Andrade ${ }^{25}$ quien reporto que $89.6 \%$ de los pacientes tenían un control glucémico inadecuado ( $\mathrm{HbA} 1 \mathrm{c}>7 \%$ ) y el $9.4 \%$ tenían un control glucémico medio. A la vez es diferente a Borba ${ }^{26}$ quien reporto que $76.7 \%$ presentaron un control glucémico adecuado en las personas con Diabetes.

El predominio de las personas diabéticas del Policlínico Manuel Manrique Nevado que no llegan a controlarse la glucosa resulta que tenga alteración del control glucémico sin darse cuenta lo que trae consigo resultados elevados en la hemoglobina glicosilada además puede afectar la función de captar mejor la glucosa lo que llega a producir modificaciones. El autocuidado de los niveles de glucosa es un comportamiento positivo para el control de la glucosa en las personas diabéticas. La Diabetes Mellitus tipo 2 se determina por la resistencia a la insulina y una decadencia gradual de las células beta dando que las personas tengan los niveles altos de glicemia ${ }^{21}$.

En cuanto al autocuidado de los pies de las personas diabéticas se encontró que $96.4 \%$ se llegan a lavarse los pies diariamente y de revisarlos cuidadosamente luego el $3.6 \%$ sin autocuidado. Los resultados encontrados fueron similares a los reportados por Tuyo ${ }^{27}$ quien reporta $62 \%$ tuvo un autocuidado en el lavado diario de pies, secado y revisión diaria mientras 
que $38 \%$ tuvieron menos práctica de autocuidado y son similares a los reportados por León ${ }^{7}$ quien reportó $78 \%$ autocuidados podológicos correctos y $22 \%$ autocuidados podológicos incorrectos. Los resultados son disimiles a los reportados por Balcázar ${ }^{28}$ quien reporta que $68.3 \%$ presentó nivel inadecuado de autocuidado mientras que el $31.7 \%$ presentó un nivel adecuado de autocuidado de los pies.

En la muestra estudiada refiere que el autocuidado de los pies es favorable en las personas con Diabetes Mellitus tipo 2 ya que por el temor de desarrollar alguna ulcera en el pie lleguen a la amputación. El autocuidado de los pies consiste en la revisión correcta ya que llegan estas personas a perder la sensibilidad y no se dan cuenta que pueden aparecer fácilmente las infecciones lo que provoca niveles altos de la glucosa. Todas las personas diabéticas deben comprobar cambios de coloración, heridas visibles o la perdida de la sensibilidad ${ }^{28}$.

En cuanto al autocuidado frente a la medicación de las personas diabéticas se encontró 94\% consumen antidiabéticos orales todos los días después de cada comida. Los resultados fueron similares a los reportados por Gonzales ${ }^{29}$ quien reporta que $86 \%$ de personas diabéticas consumen Metformina todos los días. Igualmente, los resultados fueron similares a los reportados por Pascacio ${ }^{30}$ quien reporta que $72.5 \%$ cumplieron su tratamiento farmacológico con Metformina y Glibenclamida y $27.5 \%$ no cumplieron con los medicamentos indicación para la Diabetes. Así mismo fueron similares a los reportados por Galiano ${ }^{22}$ quien reporto que $85.9 \%$ de las personas con Diabetes llevaron un régimen medicamentoso adecuado y $14.1 \%$ no toman sus antidiabéticos orales.

El predominio de las personas con Diabetes del Policlínico Manuel Manrique Nevado si llegan a consumir regularmente los antidiabéticos orales indicados ya que el tratamiento farmacológico en las personas diabéticas favorece a la persona que padece esta enfermedad controlando de manera considerable los niveles de glucosa al inicio del proceso de la Diabetes. La necesidad de entrar en tratamiento farmacológico se da porque no hay sido suficiente el control de peso y ejercicio durante un perdido razonable o bien existan signos de descontrol importantes en la persona. En las personas encuestadas, el mayor porcentaje consumen antidiabéticos orales estos tienen la función de corregir el descontrol de la Diabetes de tal manera que a largo plazo debe funcionar la alimentación saludable, la actividad física y el control de peso aplicados correctamente ${ }^{31}$.

En cuanto al autocuidado frente al consumo de tabaco en las personas diabéticas se encontró que $79.8 \%$ si llegaron a consumir tabaco durante su enfermedad. Los resultados encontrados en la presente investigación en el autocuidado frente al tabaquismo fueron disimilares a los reportados por Zambrano ${ }^{14}$ reportaron que $97.3 \%$ no son fumadores activos y $2.7 \%$ si son fumadores activos. Además, son similares a los reportados por Amaral ${ }^{20}$ quien reporto que $95.9 \%$ son fumadores activos y $4.1 \%$ han dejado de fumar. Además, son disimiles a los reportados por Ausili ${ }^{32}$ quien reporto que $11.9 \%$ son personas diabéticas fumadores y $88.1 \%$ de estas personas no son fumadores.

El predominio de las personas diabéticas del Policlínico Manuel Manrique Nevado son fumadores activos dando que el tabaquismo dentro del día a día de una persona con Diabetes Mellitus es un factor de alto riesgo de contraer enfermedades cardiovasculares ocasione constricción de los pequeños vasos sanguíneos que conducen a complicaciones. El tabaquismo y la Diabetes Mellitus tipo 2 tiene una estrecha relación ya que es un elemento importante en el desarrollo y aparición de múltiples complicaciones donde tienen 2 a 4 veces más a sufrir una enfermedad cardiaca, trombosis y neuropatía frente a los diabéticos no fumadores ${ }^{33}$. 
En la tabla $N^{\circ} 04$ se puede observar que existe correlación entre la Funcionalidad familiar y el autocuidado en medicamentos de las personas con Diabetes Mellitus tipo 2 donde. Los resultados difieren a los encontrados por Condori ${ }^{34}$ en su investigación realizada en Arequipa quien reporto que si existe relación significativa entre las variables de autocuidado en relación al tratamiento farmacológico $(p=0.062)$ además la investigación reportada por León ${ }^{35}$ donde se encontró relación significativa entre el funcionamiento familiar y el tratamiento farmacológico $(p=0.078)$. En los resultados reportados por Zenteno ${ }^{36}$ quien reporto que existe relación significativa funcionamiento familiar medio y la adherencia al tratamiento de la persona diabética $(p=0.058)$

El funcionamiento familiar está constituido sobre la base de socialización, cohesión, apoyo y regulación de sus miembros e incluye mecanismos que posibilitan auto integrarse y modificarse donde este es la matriz del desarrollo psicosocial de la persona diabética que debe adaptarse a la sociedad y posibilitar la continuidad de su autocuidado. ${ }^{9}$ Es decir que la funcionalidad familiar y la Diabetes pueden influirse entre ellas ya que esta enfermedad crónica altera la vida familiar e impone nuevos y permanentes cambios en los estilos de vida ${ }^{37}$.

Para lograr una buena funcionalidad familiar se necesita que todos los integrantes de la familia se incluyan en el plan de tratamiento de la persona con Diabetes por lo que se requiere que la familia participe en los programas educativos. Hay ocasiones que esta adaptabilidad se da incorrectamente en el sistema familiar lo que aparece un desequilibrio familiar donde la persona percibe a sus familias como conflictivas o desorganizadas ante una situación ${ }^{14}$.

\section{Conclusiones}

En la presente investigación se encontró una asociación significativa entre la funcionalidad familiar y el autocuidado específicamente relacionado con la adherencia al tratamiento farmacológico de las personas diabéticas que participaron del estudio (valor de $p=0.034$ ).

El $52.4 \%$ de las personas con Diabetes Mellitus entrevistadas corresponde a la funcionalidad familiar de rango medio y fue clasificada como no saludable. Lo cual implica que estas familias tienen tendencia a la disfuncionalidad generada por el mayor predominio de la cohesión familiar que de la adaptabilidad, por tanto, es muy difícil que los integrantes de la familia logren enfrentar el desajuste que representa para la familia el que uno de sus integrantes sea una persona diabética.

El $86.9 \%$ de las personas adultas entrevistadas fueron catalogados como "sin prácticas de autocuidado" en la Diabetes debido a que llevan comportamiento como el consumo de productos altos en carbohidratos y azucares, la falta de ejercicio, desconocimiento de los controles de glucosa y falta de concientización sobre el consumo del cigarrillo.

\section{Referencias}

1. Diabetes: países con mayor número de afectados 2017 Mundial [Internet]. Statista. 2017 [Citado 17 Jun 2018]. Disponible en: https://es.statista.com/estadisticas/636099/paises-con-mayornumero-de-adultos-con-diabetes-a-nivel-mundial/

2. Organización Mundial de la Salud. Informe Mundial sobre la Diabetes [Internet]. Ginebra; 2016 [Citado 11 Sept 2018]. Disponible en: www.who.int

3. Han N, Kirigia J, Claude J, Ogurstova K, Guariguata L, Rathmann W, et al. Diabetes Atlas de la FID [Internet]. Vol. 8, Federación Internacional de Diabetes. 2017 [Citado 11 Sept 2018]. Disponible en: www.diabetesatlas.org 
4. Asociación Americana de Diabetes. Clasificación y diagnóstico de la diabetes [Internet]. Vol. 39, Diabetes care. American Diabetes Association; 2016 [Citado 19 Sept 2018]. p. S13-22. Disponible en: http://www.ncbi.nlm.nih.gov/pubmed/26696675

5. Aguilar V. Apoyo familiar en adultos mayores que asisten al programa de Diabetes de un hospital del MINSA en Lima.2017 [Tesis de licenciatura en Internet]. Lima: Universidad Nacional Mayor de San Marcos; 2018 [Citado 21 Sept 2018]. Disponible en: http://cybertesis.unmsm.edu.pe/bitstream/handle/cybertesis/7705/Aguilar_mv.pdf?sequence=1\&i sAllowed $=y$

6. Campos A, Vicente D. Perfil Clínico Epidemiológico de Diabetes Mellitus Tipo 2, en pacientes atendidos en los Hospitales Regional Docente Las Mercedes y Provincial Docente Belén Lambayeque 2015 - 2016 [Tesis de licenciatura en Internet]. Lambayeque: Universidad Nacional Pedro Ruiz Gallo. Universidad Nacional Pedro Ruiz Gallo; 2017 [Citado 17 Jun 2018]. Disponible en: http://repositorio.unprg.edu.pe/handle/UNPRG/951

7. León M. Autocuidados Podológicos en adultos diabéticos del Policlínico Chiclayo Oeste,2016 [Tesis de licenciatura en Internet]. Chiclayo: Universidad de San Martín de Porres; 2018 [Citado 14 Sept 2018]. Disponible

en: http://www.repositorioacademico.usmp.edu.pe/bitstream/usmp/3220/3/leon_qmn.pdf

8. Noxpanco I, González M, Crespo S. Salud-competencia familiar en el autocuidado en pacientes con Diabetes Mellitus tipo 2. Rev Enf Univ. 2007; 4(3): 27-32

9. Losada A. Familia y Psicología [Internet]. ed. Buenos Aires: Dunken; 2015 [Citado 19 Oct 2018]. 208 https://books.google.com.pe/books?id=dj8gCAAAQBAJ\&printsec $=$ frontcover\&dq=funcionalidad $+f$ amiliar+y+psicologia\&hl=es-419\&sa=X\&ved=0ahUKEwi2y67g6pLeAhXPqlkKHYyXBEQ6AEIMzAC\# $\mathrm{v}=$ onepage\& $q=$ funcionalidad familiar y psicologia\& $f=$ false

10. Hernández R, Fernández C, Baptista M. Metodología de la Investigación. 6.a ed. Mexico:Interamericana, McGraw-Hill; 2014. 634 p.

11. Ponce $E$, Gómez $F$, Terán M, Irigoyen A, Landgrave $S$. Validez de constructo del cuestionario FACES III en español (México). Atención Primaria. 2002;30(10): 624-30

12. 12. Caro J, Morilla JC, Villa F, Cuevas M, Lupiáñez I, Morales JM. Adaptación cultural al español y validación psicométrica del Summary of Diabetes Self-Care Activities measure (SDSCA) en personas con diabetes mellitus tipo 2. Rev Aten Primaria [Internet]. 2016 [Citado 12 Sept 2018]; 48(7): 45867. Disponible en: www.elsevier.es/aphttp://dx.doi.org/10.1016/j.aprim.2015.08.0050212-6567/

13. Vásquez J. Cohesión, adaptabilidad familiar y tipo de familia su relación en el control de la diabetes del adulto mayor, Pasaje 2016. [Tesis de licenciatura]. Ecuador: Universidad Nacional de Loja; 2017 [Citado 2 Oct 2018]. Disponible http://dspace.unl.edu.ec/jspui/bitstream/123456789/18298/1/JORGE VICENTE VASQUEZ ILLESCAS.pdf

14. Zambrano D, Carlos J. Influencia de la funcionalidad familiar sobre el autocuidado en pacientes diabéticos tipo 2 atendidos en el centro de salud "El Carmen" durante el periodo de Septiembre a Noviembre del 2016 [Tesis de licenciatura en Internet]. Ecuador: Pontificia Universidad Católica del Ecuador; 2016 [Citado 12 Sept 2018]. Disponible en: http://repositorio.puce.edu.ec/bitstream/handle/22000/12858/TESISDRLOORYZAMBRANO.pdf?seq uence $=1$ \&isAllowed $=y$

15. Moraga R, Cid P. Factores biopsicosociales que se relacionan con el funcionamiento de las familias con pacientes dependientes. Cienc y Enfermería [Internet]. 2017 [Citado 27 May 2019]; 23(2): 5767. Disponible en: https://scielo.conicyt.cl/pdf/cienf/v23n2/0717-9553-cienf-23-02-00057.pdf

16. Ortiz D. La terapia familiar sistémica [Internet]. ed. Quito: Abya-Yala; 2008 [Citado 24 Oct 2018]. 334 p. Disponible en: https://dspace.ups.edu.ec/bitstream/123456789/6096/1/Terapia familiar sistemica.pdf

17. Olson D, Craddock A. Modelo circumplejo de sistemas matrimoniales y familiares. Rev Fam Ther [Internet]. 2000 [Citado 14 Sept 2018]; 1(2): 144-67. Disponible en: https://onlinelibrary.wiley.com/doi/pdf/10.1111/1467-6427.00144

18. Del Pezo E. Participación familiar en el autocuidado del paciente diabético "Centro de salud \#1 Santa Elena" [Tesis de licenciatura en Internet]. Ecuador: Universidad Estatal Península de Santa Elena; 
2013 [Citado $14 \quad$ Sept 2018]. Disponible en: http://repositorio.upse.edu.ec/bitstream/46000/992/1/ELOISA DEL PEZO-TESIS.pdf

19. León $E$, Orozco L, Argüelles V, Hernández L, Luzanía M, Campos Y. La alfabetización en salud como factor clave en el autocuidado de la dieta en personas con Diabetes Mellitus tipo 2. Univ y Salud [Internet]. 2019 [Citado 19 Sept 2019]; 21(2): 132-40. Disponible en: http://revistas.udenar.edu.co/index.php/usalud/article/view/3860

20. Amaral R, Barbosa A, Teixeira C, Brandão L, Afonso T, Bezerra A, et al. Conocimiento de los diabéticos sobre la enfermedad y el autocuidado. Rev enferm [Internet]. 2019 [Citado 19 Sept 2019]; 2(13): 346-52. Disponible en: https://pesquisa.bvsalud.org/portal/resource/pt/biblio1009997

21. Islas S, Revilla C. Diabetes mellitus: actualizaciones [Internet]. Ed. Mexico: Alfilr; 2013 [Citado 5 Oct 2018]. 424 p. Disponible en: www.amc.org.mx

22. Galiano M, Calvo M, Feito M, Aliaga M, Leiva S, Mujica B. Condición de salud de pacientes diabéticos y su satisfacción con el tratamiento para la enfermedad. Cienc y Enfermería [Internet]. 2013 [Citado 19 Sept 2019]; 19(2): 57-66. Disponible en: https://www.redalyc.org/articulo.oa?id=370441813006

23. Jaimes K, Blancas L. Autocuidado en pacientes con Diabetes Mellitus tipo II en el hospital nacional Sergio E. Bernales, Comas-2018 [Tesis de licenciatura en Internet]. Lima: Universidad César Vallejo; 2018 [Citado 19 Sept 2019]. Disponible en: http://repositorio.ucv.edu.pe/bitstream/handle/UCV/18105/Jaimes_CK.pdf?sequence=1\&isAllowed $=\mathrm{y}$

24. Pérez Z, Rivas V, Martínez A. Percepción del apoyo familiar y dificultades relacionadas con la diabetes en el adulto mayor. Horiz Sanit [Internet]. 2015 [Citado 26 May 2019]; 14(1): 14-20. Disponible en: http://www.redalyc.org/articulo.oa?id=457844964003

25. Andrade C, Ribeiro G, Santos C, Neves R, Moreira E. Factores asociados con altos niveles de hemoglobina glucosilada en pacientes con Diabetes. BM] Open [Internet].2017 [Citado 19 Sept 2019]; 7(12): 1-10. Disponible en: http://www.ncbi.nlm.nih.gov/pubmed/29247092

26. Borba A, Arruda I, Marques A, Leal M, Diniz A. Conocimiento y actitud sobre el autocuidado de la diabetes en adultos en atención primaria de salud. Cien Saude Colet [Internet]. enero de 2019 [Citado 19 Sept 2019]; 24(1): 125-36. Disponible en: http://www.scielo.br/scielo.php?script=sci_arttext\&pid=S1413-

$81232019000100125 \&$ lng $=$ pt\&tlng $=$ pt

27. Tuyo K. Nivel de conocimientos y su relación con la práctica del autocuidado en adultos con Diabetes Mellitus tipo II, en el C. S. San Francisco [Internet]. Tacna: Universidad Nacional Jorge Basadre Grohmann; 2015 [Citado 12 Sept 2018]. Disponible en: http://repositorio.unjbg.edu.pe/bitstream/handle/UNJBG/2175/729_2015_tuyo_poma_kl_facs_enf ermeria.pdf?sequence $=1 \&$ isAllowed $=y$

28. Balcázar M, Escate Y, Choque C, Velásquez D. Capacidades y actividades en el autocuidado del paciente con pie diabético. Rev enferm Herediana [Internet]. 2014 [Citado 14 Sept 2018]; 7(2): 638. Disponible en: https://faenf.cayetano.edu.pe/images/pdf/Revistas/2014/julio/Articulo 2-7-2.pdf

29. González O. Adherencia Terapéutica y Funcionalidad Familiar en pacientes con Diabetes Mellitus tipo 2 de la consulta externa de Medicina Familiar en el centro médico «Lic. Adolfo López Mateos» [Tesis de licenciatura en Internet]. México: Universidad Autónoma del estado de México; 2013 [Citado $12 \quad$ Sept 2018]. 12 Disponible http://ri.uaemex.mx/bitstream/handle/20.500.11799/14302/405580.pdf?sequence=1\&isAllowed= $y$

30. Pascacio G, Ascencio G, Cruz A, Guzmán C. Adherencia al tratamiento y conocimiento de la enfermedad en pacientes con Diabetes Mellitus tipo 2. Salud en Tabasco [Internet]. 2016 [Citado 19 Sept 2019]; 22(1-2): 23-31. Disponible en: https://www.redalyc.org/articulo.oa?id=48749482004

31. Estela B, Álvarez C, Molina V, Lara A, Fuentes L, Barriguete A. Diabetes: Guía para pacientes [Internet]. 2.a ed. CENAPRECE. México: Pressprinting; 2011 [Citado 20 Sept 2019]. p. 48. Disponible en: http://www.cenaprece.salud.gob.mx/programas/interior/adulto/descargas/pdf/GuiaPacientesDiabe tes.pdf 
32. Ausili D, Bulgheroni M, Ballatore $P$, Specchia C, Ajdini A, Bezze $S$, et al. Autocuidado, calidad de vida y resultados clínicos de pacientes con Diabetes tipo 2: un estudio transversal observacional. Acta Diabetol [Internet]. 2017 [Citado 19 Sept 2019];54(11):1001-8. Disponible en: http://link.springer.com/10.1007/s00592-017-1035-5

33. Gómez C, Palma S. Manual práctico de Nutrición y salud. 2016 [Citado 20 Sept 2019]. p. 296-310. Disponible

en: https://www.kelloggs.es/content/dam/europe/kelloggs_es/images/nutrition/PDF/Manual_Nutricion Kelloggs_Capitulo_19.pdf

34. Condori S, Cruz G. Funcionalidad familiar y Adherencia al tratamiento de diabetes mellitus tipo 2, Pacientes Hospital Regional Honorio Delgado [tesis de licenciatura]. Arequipa: Universidad Nacional de San Agustín de Arequipa; 2018 [Citado 12 Sept 2018]. Disponible en: http://repositorio.unsa.edu.pe/bitstream/handle/UNSA/5780/ENcofus.pdf?sequence=1\&isAllowed $=\mathrm{y}$

35. León C, Ehlerman C. Funcionalidad familiar en pacientes con Diabetes Mellitus tipo 2 en Hospital ISSS Sonsonate [Tesis de licenciatura]. El Salvador: Universidad de El Salvador; 2015 [Citado 5 Oct 2019]. Disponible en: http://ri.ues.edu.sv/9964/1/TESIS FUNCIONALIDAD FAMILIAR EN PACIENTES CON DIABETES MELLITUS TIPO 2 EN HOSPITAL ISSS SONSONATE.pdf

36. Zenteno M, García G, Marín M, Flores M. Influencia del funcionamiento familiar en la adherencia al tratamiento terapéutico y el control glucémico de adultos y adultos mayores con diabetes tipo 2. Diabetes Care [Internet]. 2015 [Citado 5 Oct 2019]; 22(6): 1239-45. Disponible en: http://care.diabetesjournals.org/cgi/doi/10.2337/dc11-2103

37. Mendizábal O, Pinto B. Estructura familiar y Diabetes [Internet]. México; 2006 [Citado 6 Nov 2018]. p. 21. Disponible en: http://www.scielo.org.bo/pdf/rap/v4n2/v4n2a3.pdf

\section{Editor}

Escuela de Enfermería de la Universidad Católica Santo Toribio de Mogrovejo, Chiclayo, Perú

\section{Cómo citar este trabajo}

Campoverde-Vilanueva F, Muro-Exebio I. Funcionalidad familiar y autocuidado en personas con Diabetes Mellitus tipo 2 - Policlínico Manuel Manrique Nevado, Chiclayo 2019. Acc Cietna: para el cuidado de la salud [Internet]. 2021; 8(2): 32 - 44. Disponible en: https://doi.org/10.35383/cietna.v8i2.633

\section{Financiación}

El presente artículo no cuenta con financiación específica de agencias de financiamiento en los sectores público o privado para su desarrollo y/o publicación.

\section{Conflicto de interés}

Los autores del artículo declaran no tener ningún conflicto de intereses en su realización.

(c) Los autores. Este artículo es publicado por la Revista Acc Cietna: para el cuidado de la salud de la Escuela de Enfermería, Universidad Católica Santo Toribio de Mogrovejo.

Este es un artículo de acceso abierto, distribuido bajo los términos de la Licencia Creative Commons Atribución-NoComercial-CompartirIgual 4.0 Internacional (CC BY-NC-SA 4.0), que permite el uso no comercial, distribución y reproducción en cualquier medio, siempre que la obra original sea debidamente citada. 